\title{
Geometrically Distinct Solutions for a class of quasilinear Schrö dinger equation with critical growth
}

\author{
Xiumei He $\mathrm{H}^{1, \text { a }}$, Xingping $\mathrm{Li}^{2, \mathrm{~b}}$ \\ ${ }^{1}$ Department of Mathematics and Statistics, Yunnan University, Kunming,650091,China \\ ${ }^{1}$ Department of Mathematics, Kunming University, Kunming, Yunnan, 650214, China \\ ${ }^{2}$ Department of Mathematics and Statistics, Yunnan University, Kunming,650091,China \\ aemail:hexiumei2004@163.com, bemail: Ixp551@aliyun.com
}

Keywords: quasilinear Schr $\ddot{O}$ dinger equation, critical growth, geometrically distinct solutions, perturbation approach.

Abstract. In this paper, we consider the quasilinear Schr $\ddot{0}$ dinger equation with critical growth $-\Delta u-\lambda u \Delta u^{2}+V(x) u=|u|^{22^{*}-2} u+g(x, u), \quad x \in \square^{N}$. We employ the perturbation approach developed by Xiangqing Liu [9] and the generalized linking approach and obtain the infinitely many geometrically distinct solutions.

\section{Introduction and Preliminaries}

In this paper, we study the following quasilinear Schrö dinger equation with critical growth

$$
-\Delta u-\lambda u \Delta u^{2}+V(x) u=|u|^{22^{*}-2} u+g(x, u), \quad x \in \square^{N},
$$

where $\lambda \geq 0,2^{*}=\frac{2 N}{N-2}(N \geq 3), g \in C\left(\square^{N} \times \square, \square\right)$ and $V \in C\left(\square^{N}, \square\right)$, whose weak variational formulation is to look for $u \in H^{1}\left(\square^{N}\right) \cap L^{\infty}\left(\square^{N}\right)$ such that

$$
\int_{\square^{N}} \nabla u \nabla \varphi d x+2 \lambda \int_{\square^{N}}\left(u^{2} \nabla u \nabla \varphi+|\nabla u|^{2} u \varphi\right) d x+\int_{\square^{N}} V(x) u \varphi d x-\int_{\square^{N}}|u|^{22^{*}-2} u \varphi d x-\int_{\square^{N}} g(x, u) \varphi d x=0
$$

for all $\varphi \in H^{1}\left(\square^{N}\right) \bigcap L^{\infty}\left(\square^{N}\right)$.

When $\lambda=1$, the following quasilinear Schr $\ddot{o}$ dinger equation

$$
-\Delta u+V(x) u-\Delta\left(u^{2}\right) u=g(x, u), \quad x \in \square^{N},
$$

has been studied recently by several authors, see [2,3,6] and the refferences therein. Solutions of equation (1.2) are standing waves the following quasilinear Schr $\ddot{o}$ dinger equation of the form

$$
i \psi_{t}+\Delta \psi-V(x) \psi+k \Delta\left(\alpha\left(|\psi|^{2}\right)\right) \alpha^{\prime}\left(|\psi|^{2}\right) \psi+g(x, \psi)=0, \quad x \in \square^{N},
$$

where $V(x)$ is a given potential, $k$ is a real constant, $\alpha$ and $g$ are real functions. The quasilinear Schrö dinger equations (1.3) are derived as models of several physical phenomena, such as see[8, 11]. It begins with [10] for the studies on mathematics. Several methods can be used to solve the equation (1.2), such as, the existence of a positive ground state solution has been studied in [14] by using a constrained minimization argument; the problem is transformed to a semilinear one in [1, 2] by a change of variables; Nehari method is used to get the existence results of ground state solutions in [12]. In [4], by a dual approach (precisely, a change of variables), an existence theorem for infinitely many periodic orbits of solutions for the equation (1.2) was obtained.

In this paper, our aim is to search the existence of infinitely many pairs of geometrically distinct solutions for problem (1.1) via the perturbation approach due to [9].

We need the following several notations. Let $H^{1}\left(\square^{N}\right):=\left\{u \in L^{2}\left(\square^{N}\right): \nabla u \in L^{2}\left(\square^{N}\right)\right\}$ with the inner product $\langle u, v\rangle_{H^{1}}=\int_{\square^{N}}(\nabla u \nabla v+u v) d x$ and the norm $\|u\|_{H^{1}}^{2}=\int_{\square^{N}}\left(|\nabla u|^{2}+|u|^{2}\right) d x$. Let the following 
assumption $(V)$ hold:

(V) $V \in C\left(\square^{N}, \square\right), 0<V_{0}:=\inf _{x \in \square^{N}} V(x)$ and $V\left(x_{1}, \cdots, x_{N}\right)$ is 1-periodic in $x_{i}, i=1, \cdots, N$.

Set $E=H^{1}\left(\square^{N}\right) \cap W^{1,4}\left(\square^{N}\right)$ with the norm $\|u\|_{E}=\|u\|_{H^{1}}+\|u\|_{W^{1,4}\left(\square{ }^{N}\right)}$. Then $E$ is a separable reflexive Banach space. The following embeddings are continuous: $E \rightarrow L^{s}\left(\square^{N}\right), s \in\left[2,22^{*}\right]$, where we denote by $\|\cdot\|_{s}$ the norm of $L^{s}\left(\square^{N}\right)$. The following embeddings are compact: $E \rightarrow L^{s}\left(\square^{N}\right), s \in\left[2,22^{*}\right)$.

Moreover, we need the following assumptions:

$\left(g_{1}\right)$ Let $g \in C\left(\square^{N} \times \square, \square\right), g\left(x_{1}, \cdots, x_{N}, s\right)$ be1-periodic in $x_{i}, 1 \leq i \leq N, g(x, s)$ is odd in $s$.

$\left(g_{2}\right)$ there exists $4<p<22^{*}$ such that $|g(x, t)| \leq C\left(1+|t|^{p-1}\right)$ for all $(x, t) \in \square^{N} \times \square$.

$\left(g_{3}\right) g(x, t)=\circ(|t|)$ uniformly in $x$ as $|t| \rightarrow 0$ and $\operatorname{tg}(x, t) \geq 0$ for all $(x, t) \in \square^{N} \times \square$.

$\left(g_{4}\right) \lim _{\mid t \rightarrow+\infty} \frac{G(x, t)}{t^{4}}=+\infty$ uniformly in $x \in \square^{N}$, where $G(x, t):=\int_{0}^{t} g(x, s) d s$.

$\left(g_{5}\right) \operatorname{tg}(x, t)-4 G(x, t) \geq \operatorname{stg}(x, s t)-4 G(x, s t), \forall(x, t) \in \square^{N} \times \square, \forall s \in[0,1]$.

The equation (1.1) is the Euler-Lagrange equation of the energy functional

$$
J(u)=\frac{1}{2} \int_{\square N}|\nabla u|^{2} d x+\frac{1}{2} \int_{\square_{N}}\left(V(x) u^{2}+2 \lambda u^{2}|\nabla u|^{2}\right) d x-\frac{1}{22^{*}} \int_{\square^{N}}|u|^{22^{*}} d x-\int_{\square^{N}} G(x, u) d x .
$$

For $\theta \in(0,1]$, let $J_{\theta}(u)=\frac{1}{4} \theta \int_{\square N}\left(|\nabla u|^{4}+u^{4}\right) d x+J(u)$. Similar to Lemma 2.1 in [5] we can proof that $J$ and $J_{\theta} \in C^{1}(E, \square)$.

Let $*$ denote the action of $Z^{N}$ on $H^{1}\left(\square^{N}\right)$ given by $(k * u)(x):=u(x-k), k \in Z^{N}$. Set $\mathrm{O}(u):=\left\{k * u: k \in Z^{N}\right\} . \mathrm{O}(u)$ is called the orbit of $u$ with respect to the action of $Z^{N}$. Under the assuming conditions, if $u$ is a solution of (1.1), then so is $k * u$ for all $k \in Z^{N}$. If $u_{0} \in H^{1}\left(\square^{N}\right) \bigcap W^{1,4}\left(\square^{N}\right)$ is a critical point of a functional $F$ and $F$ is $Z^{N}$-invariant, i.e. $F(k * u)=F(u)$ for all $k \in Z^{N}$ and all $u \in H^{1}\left(\square^{N}\right) \bigcap W^{1,4}\left(\square^{N}\right)$, then $\mathrm{O}\left(u_{0}\right)$ is called a critical orbit of $F$. Two solutions $u_{1}, u_{2}$ of (1.1) are said to be geometrically distinct if $\mathrm{O}\left(u_{1}\right) \neq \mathrm{O}\left(u_{2}\right)$.

A sequence $\left\{u_{n}\right\} \subset E$ is called a P. S. sequence of $J$ if $\left\{J\left(u_{n}\right)\right\}$ is bounded and $J\left(u_{n}\right) \rightarrow 0$ in $E^{*}$. We say that $J$ satisfies the P. S. condition if every P. S. sequence possesses a convergent subsequence.

The main result of this paper is the following.

Theorem 1.1 Suppose that $(V)$ and $\left(g_{1}\right)-\left(g_{5}\right)$ are satisfied. Then the equation (1.1) admits infinitely many pairs $\pm u$ of geometrically distinct solutions.

Remark 1.1 For the case of non-critical growth, notice that $\left(g_{5}\right)$ is weaker than the following $\left(g_{5}\right)^{\prime}$. Hence, our Theorem 1.1, at the case $\lambda=1$, improves Theorem1.1 in[4], and our method is different from[4]. $\left(g_{5}\right)^{\prime} \frac{g(x, t)}{t^{3}}$ is non-increasing on $(-\infty, 0)$ and non-decreasing on $(0, \infty)$.

Throughout the paper, $C, c, C_{i}$ and $c_{i}$ express distinct constants.

\section{The proof of the main result}

To begin with, we define, for each fixed $\theta \in(0,1], M=M(\theta):=\left\{u \in E \backslash\{0\}:\left\langle J_{\theta}{ }^{\prime}(u), u\right\rangle=0\right\}$. For any $u \in E \backslash\{0\}$ and $t>0$, set $h(t):=J_{\theta}(t u)$.

We divide the proof of Theorem 1.1 into the following Lemmas.

Lemma 2.1 For each $u \in E \backslash\{0\}$, there exists an unique $t_{u}>0$ such that $h\left(t_{u}\right)=\underset{s \geq 0}{\max } h(s)$, 
$h^{\prime}(t)>0$ for $0<t<t_{u}$ and $h^{\prime}(t)<0$ for $t_{u}<t$. Moreover, tu $\in M$ if and only if $t=t_{u}$.

Proof. For any $\varepsilon>0$, by $\left(g_{2}\right)$ and $\left(g_{3}\right)$, there exists $C_{\varepsilon}>0$ such that

$$
|g(x, u)| \leq \varepsilon|u|+C_{\varepsilon}|u|^{p-1},|G(x, u)| \leq \varepsilon|u|^{2}+C_{\varepsilon}|u|^{p}, \forall(x, u) \in \square^{N} \times \square .
$$

Hence $h$ has a positive maximum and there exist a $t_{u}>0$ such that $h^{\prime}\left(t_{u}\right)=0$ and $h^{\prime}(t)>0$ for $0<t<t_{u}$.

We claim that $h^{\prime}(t) \neq 0$ for all $t>t_{u}$. Indeed, if the conclusion is false, then, from the above arguments, there exists a $t_{u}<t_{2}<+\infty$ such that $h^{\prime}\left(t_{2}\right)=0$ and $h\left(t_{u}\right) \geq h\left(t_{2}\right)$. But $\left(g_{5}\right)$ implies that $h\left(t_{2}\right)>\frac{1}{4} t_{u}^{2} \int_{\square}\left[|\nabla u|^{2}+V(x) u^{2}\right] d x+\frac{1}{2 N} t_{u}^{22^{*}} \int_{\square N}|u|^{22^{*}} d x+\int_{\square}\left[\frac{1}{4} g\left(x, t_{u} u\right) t_{u} u-G\left(x, t_{u} u\right)\right] d x=h\left(t_{u}\right)$.

This is a contradiction. This claim is proved.

The second conclusion is an immediate consequence of the fact that $h^{\prime}(t)=t^{-1}\left\langle J_{\theta}{ }^{\prime}(t u), t u\right\rangle$. This completes the proof of Lemma 2.1.

Lemma 2.2 (1)There exists a $\rho>0$ such that $c(\theta):=\inf _{u \in M} J_{\theta}(u) \geq \inf _{u \in S_{\rho}} J_{\theta}(u)>0$ and there exists a constant $\delta_{0}>0$ such that $\|u\|_{E} \geq \delta_{0}$ for all $u \in M$, where $S_{\rho}:=\left\{u \in E:\|u\|_{E}=\rho\right\}$.

(2)There exists a positive number $\delta_{1}$ independent of $\theta$ such that $c(\theta) \geq \delta_{1}$.

Proof. For any $\rho>0$, if $u \in E$ with $\|u\|_{E} \leq \rho$, then $\int_{\square^{N}}\left|u^{2}\right||\nabla u|^{2} d x \leq \rho^{4}$. Hence for small $\rho>0$ and $\varepsilon<\frac{V_{0}}{4}$, by $(V),\left(g_{2}\right),\left(g_{3}\right)$ and the Sobolev inequality, one has

$$
\begin{aligned}
J_{\theta}(u) \geq & \frac{\theta}{4} \int_{\square N}\left(|\nabla u|^{4}+u^{4}\right) d x+\frac{1}{2} \int_{\square^{N}}\left(1+2 \lambda u^{2}\right)|\nabla u|^{2} d x+\frac{1}{2} \int_{\square N} V(x) u^{2} d x \\
& -\frac{1}{22^{*}} \int_{\square N}|u|^{22^{*}} d x-\int_{\square N}\left(\varepsilon u^{2}+C_{\varepsilon}|u|^{22^{*}}\right) d x \geq \frac{\theta}{4}\|u\|_{W^{1,4}}^{4}+C_{2}\|u\|_{H^{1}}^{2} \geq C(\theta)\|u\|_{E}^{4}
\end{aligned}
$$

Whenever $\|u\|_{E} \leq \rho$. For any $u \in M$, Lemma 2.1 implies that $J_{\theta}(u)=\max _{t \geq 0} J_{\theta}(t u)$. Take a $s>0$ with $s u \in S_{\rho}$. Then $J_{\theta}(u) \geq J_{\theta}(s u) \geq \inf _{v \in S_{\rho}} J_{\theta}(v) \geq C(\theta) \rho^{4}>0$ and hence $c(\theta):=\inf _{u \in M} J_{\theta}(u) \geq \inf _{v \in S_{\rho}} J_{\theta}(v)>0$. Moreover, for each $u \in M$, by $\left(g_{3}\right)$, one has $c(\theta) \leq C_{3}\left(\|u\|_{E}^{4}+\|u\|_{E}^{2}\right)$. Hence there exists a constant $\delta_{0}>0$ such that $\|u\|_{E} \geq \delta_{0}$ for all $u \in M$.

Further, for any $\rho>0$, if $u \in E$ with $\alpha(u):=\|u\|_{H^{1}}+\left(\int_{\square^{N}} u^{2}|\nabla u|^{2} d x\right)^{\frac{1}{4}} \leq \rho$, then $\int_{\square^{N}} u^{2}|\nabla u|^{2} d x \leq \rho^{4}$. Hence for small $\rho_{0}>0$ and $\varepsilon<\frac{V_{0}}{4}$, by $(V),\left(g_{2}\right),\left(g_{3}\right)$ and the Sobolev inequality, one has $J_{\theta}(u) \geq C\left(\|u\|_{H^{1}}^{2}+\int_{\square^{N}} u^{2}|\nabla u|^{2} d x\right)$ whenever $\alpha(u) \leq \rho_{0}$. For any $u \in M$, Lemma 2.1 implies that $J_{\theta}(u)=\max _{t \geq 0} J_{\theta}(t u)$. Take a $s>0$ with $\alpha(s u)=\rho_{0}$. Then $J_{\theta}(u) \geq J_{\theta}(s u) \geq \frac{C}{2} \rho_{0}^{4}:=\delta_{1}>0$, hence $c(\theta) \geq \delta_{1}$. This completes the proof of Lemma 2.2.

Lemma $2.3 J_{\theta}$ is coercive on $M$,i.e. $J_{\theta}(u) \rightarrow+\infty$ as $\|u\|_{E} \rightarrow \infty, u \in M$.

Proof. Arguing by contradiction, suppose there exists a sequence $\left\{u_{n}\right\} \subset M$, such that $\left\|u_{n}\right\|_{E} \rightarrow \infty$ and $J_{\theta}\left(u_{n}\right) \leq d$ for some $d<0$. By $\left(g_{5}\right)$ and $(V)$, one has

$$
d \geq J_{\theta}\left(u_{n}\right)-\frac{1}{4}\left\langle J_{\theta}^{\prime}\left(u_{n}\right), u_{n}\right\rangle \geq \frac{1}{4} \min \left\{1, V_{0}\right\} \int_{\square N}\left(\left|\nabla u_{n}\right|^{2}+\left|u_{n}\right|^{2}\right) d x+\frac{1}{2 N} \int_{\square N}\left|u_{n}\right|^{22^{*}} d x .
$$

Hence $\left\{\left\|u_{n}\right\|_{H^{1}}\right\}$ and $\left\{\left\|u_{n}\right\|_{L^{22^{*}}\left(R^{N}\right)}\right\}$ are bounded. By interpolation, $\left\{u_{n}\right\}$ is bounded in $L^{s}\left(\square{ }^{N}\right), 2 \leq s \leq 22^{*}$. 
Consequently, by (2.1), there exists a constant $C_{0}>0$ such that $\left|\int_{\square^{N}} G\left(x, u_{n}\right) d x\right| \leq C_{0}$, hence $J_{\theta}\left(u_{n}\right) \geq \frac{\theta}{4}\left\|u_{n}\right\|_{W^{1,4}}^{4}-C$, which implies $\frac{\theta}{4}\left\|u_{n}\right\|_{W^{1,4}}^{4} \leq d+C<\infty$. Consequently $\left\{\left\|u_{n}\right\|_{E}\right\}$ is bounded, a contradiction. This ompletes the proof of lemma2.3.

Lemma 2.4 Let $D \subset E \backslash\{0\}$ be a compact subset. Then there exists a $R>0$ such that $J_{\theta}<0$ on $\left(\square^{+} D\right) \backslash B_{R}(0)$, where $B_{R}(0):=\left\{u \in E:\left\|u_{n}\right\|_{E}<R\right\}$.

Proof. Arguing by a contradiction, suppose that there exist equences $\left\{u_{n}\right\} \subset D$ and $\left\{t_{n}\right\} \subset \square^{+}$such that $J_{\theta}\left(t_{n} u_{n}\right) \geq 0$ and $t_{n} \rightarrow+\infty$ as $n \rightarrow \infty$. By the compactness of $D$, we may assume that $u_{n} \rightarrow u \in D$ and $\left\|u_{n}\right\|_{E} \leq C$. Hence $0 \leq \frac{J_{\theta}\left(t_{n} u_{n}\right)}{t_{n}^{4}} \leq C_{1}-\frac{1}{22^{*}} t_{n}^{2\left(2^{*}-2\right)} \int_{\square N}\left|u_{n}\right|^{22^{*}} d x \rightarrow-\infty$, a contradiction. This completes the proof of Lemma 2.4.

Let $S$ be the unit sphere in $E$. Define a mapping $m=m(\theta): S \rightarrow M$ and a functional $\Psi=\Psi(\theta): S \rightarrow \square$ by $m(u)=t_{u} u$ and $\Psi(u)=J_{\theta}(m(u))$, where $t_{u}$ is as in Lemma 2.1. By Lemma 2.1, 2.2 and 2.4, similar to Lemmas 3.6-3.8 in [4],we can prove the following Lemmas 2.5-2.7.

Lemma 2.5 The mapping $m$ is a homeomorphism between $S$ and $M$, and the inverse of $m$ is given by $m^{-1}(u)=\frac{u}{\|u\|_{E}}$.

Lemma 2.6 (1) $\Psi \in C^{1}(S, \square)$ and for each $u \in S$, one has $\left\langle\Psi^{\prime}(u), z\right\rangle=\|m(u)\|_{E}\left\langle J^{\prime}{ }_{\theta}(m(u)), z\right\rangle$ for all $z \in T_{u}(S)$, where $T_{u}(S)$ is the tangent space of $S$ at point $u$.

(2) If $\left\{u_{n}\right\}$ is a Palais-Smale sequence of $\Psi$, then $\left\{m\left(u_{n}\right)\right\}$ is a Palais-Smale sequence of $J_{\theta}$. If $\left\{u_{n}\right\} \subset M$ is a bounded Palais-Smale sequence of $J_{\theta}$, then $\left\{m^{-1}\left(u_{n}\right)\right\}$ is a Palais-Smale sequence of $\Psi$.

(3) $u$ is a critical point of $\Psi$ if and only if $m(u)$ is a nontrivial critical point of $J_{\theta}$. Moreover, the corresponding values of $\Psi$ and $J_{\theta}$ coincide and $\inf _{S} \Psi=\inf _{M} J_{\theta}$. (4) If $J_{\theta}$ is even, then so is $\Psi$.

Lemma 2.7 The mapping $m^{-1}$ defined in Lemma 2.5 is Lipschitz continuous.

Lemma $2.8 c_{k}(\theta)$ is a critical value of $\Psi$.

Proof. If $c_{k}(\theta)$ is not a critical value of $\Psi$, then for any $w \in S$, one has $\Psi(w) \neq c_{k}(\theta)$ or $\Psi^{\prime}(w) \neq 0$. Hence there exists $\delta>0$ such that $N_{C_{k}(\theta), \delta}:=\left\{w \in S:\left|\Psi(w)-c_{k}(\theta)\right|<\delta,\left\|\Psi^{\prime}(w)\right\|_{E^{*}}<\delta\right\}=\varnothing$. Otherwise, there exists a sequence $\left\{w_{n}\right\} \subset S$ such that $\Psi\left(w_{n}\right) \rightarrow c_{k}(\theta)$ and $\left\|\Psi^{\prime}\left(w_{n}\right)\right\|_{E^{*}} \rightarrow 0$. Set $u_{n}=m\left(w_{n}\right)$. Then, $\left\{u_{n}\right\} \subset M$ is a $(P S)_{c_{k}(\theta)}$ sequence of $J_{\theta}$. By Lemma 2.3 the sequence $\left\{u_{n}\right\}$ is bounded in $E$. Hence, up to a subsequence, one has $u_{n} \stackrel{\text { weak }}{\longrightarrow} u$ in $W^{1,4}\left(\square^{N}\right)$ and $H\left(\square^{N}\right)$. Notice that $22^{*}<\frac{4 N}{N-2}$, one has $u_{n} \rightarrow u$ in $L_{l o c}^{s}\left(\square^{N}\right)$ for all $s \in\left[2,22^{*}\right)$ and $u_{n}(x) \rightarrow u(x)$ a.e. $x \in \square^{N}$.By weakly convergence, one has $\int_{\square^{N}}\left|\nabla u_{n}\right|^{2} d x \geq 2 \int_{\square^{N}} \nabla u_{n} \cdot \nabla u d x-\int_{\square^{N}}|\nabla u|^{2} d x=\int_{\square^{N}}|\nabla u|^{2} d x+o_{n}(1)$ and $o_{n}(1)=\left\langle J_{\theta}^{\prime}\left(u_{n}\right)-J_{\theta}^{\prime}(u), u_{n}-u\right\rangle$

$$
\begin{aligned}
& \geq C \theta\left\|u_{n}-u\right\|_{W^{1,4}}^{4}+C\left\|u_{n}-u\right\|_{H^{1}}^{2}+2 \lambda \int_{\square N}\left(\left|u_{n}\right|^{2}-|u|^{2}\right) \cdot \nabla u \cdot \nabla\left(u_{n}-u\right) d x \\
& +2 \lambda \int_{\square}\left(\left|\nabla u_{n}\right|^{2}-|\nabla u|^{2}\right) \cdot u \cdot\left(u_{n}-u\right) d x-\int_{\square}\left[\left|u_{n}\right|^{22^{*}-2} u_{n}-|u|^{22^{*}-2} u\right] \cdot\left(u_{n}-u\right) d x \\
& -\int_{\square N}\left[g\left(x, u_{n}\right)-g(x, u)\right] \cdot\left(u_{n}-u\right) d x
\end{aligned}
$$

where $o_{n}(1) \rightarrow 0$ as $n \rightarrow \infty$. Hence, by Hö lder inequality and the boundedness of $\left\{u_{n}\right\}$ in $E$, one 
has

$$
C \theta\left\|u_{n}-u\right\|_{W^{1,4}}^{4}+C\left\|u_{n}-u\right\|_{H^{1}}^{2} \leq o_{n}(1)+C_{1}\left\|u_{n}-u\right\|_{22^{*}}+\int_{\square^{N}}\left(\left|g\left(x, u_{n}\right)\right|+|g(x, u)|\right) \cdot\left|u_{n}-u\right| d x .
$$

For any $\varepsilon>0$, by $\left(g_{2}\right),\left(g_{3}\right)$ and the boundedness of $\left\{u_{n}\right\}$ in $E$, there exist constants $C>0$ and

$$
C_{\varepsilon}>0 \text { such that } \int_{\square^{N}}\left(\left|g\left(x, u_{n}\right)\right|+|g(x, u)|\right)\left|u_{n}-u\right| d x \leq \varepsilon C\left\|u_{n}-u\right\|_{E}+C_{\varepsilon}\left\|u_{n}-u\right\|_{22^{*}} \text {. }
$$

Combining (2.2) and (2.3) we know that for any $\varepsilon>0$, there exist positive constants $C$ and $C_{\varepsilon}$ such that

$$
C \theta\left\|u_{n}-u\right\|_{W^{1,4}}^{4}+C\left\|u_{n}-u\right\|_{H^{1}}^{2} \leq o_{n}(1)+\varepsilon C\left\|u_{n}-u\right\|_{E}+C_{\varepsilon}\left\|u_{n}-u\right\|_{22^{*}} .
$$

If $\left\|u_{n}-u\right\|_{22^{*}}$ does not go to 0 as $n \rightarrow \infty$, then, by Lemma I.1 in [7], up to a subsequence, there exists $\varepsilon_{0}>0$ such that $\sup _{y \in \square^{N}} \int_{B_{1}(y)}\left|u_{n}-u\right|^{2} d x \geq \varepsilon_{0}$. Hence there exists $y_{n} \in \square^{N}$ such that

$\int_{B_{1}\left(y_{n}\right)}\left|u_{n}-u\right|^{2} d x=\max _{y \in \square^{N}} \int_{B_{1}(y)}\left|u_{n}-u\right|^{2} d x \geq \varepsilon_{0}$. By the assumptions of periodicity, we can assume $\left\{y_{n}\right\}$ is bounded in $\square{ }^{N}$. Consequently, there exists a bounded domain $\Omega \subset \square^{N}$ such that

$\int_{\Omega}\left|u_{n}-u\right|^{2} d x \geq \int_{B_{1}\left(y_{n}\right)}\left|u_{n}-u\right|^{2} d x \geq \varepsilon_{0}$, a contradiction. Hence $\left\|u_{n}-u\right\|_{22^{*}} \rightarrow 0$ as $n \rightarrow \infty$. Consequently, by (2.7), $u_{n} \rightarrow u$ in $E$, and hence $u$ is a critical point of $J_{\theta}$ and $J_{\theta}(u)=c_{k}(\theta)$. Moreover, by Lemma 2.7 we know that $u \in M$, and hence Lemma 2.5 implies $w:=m^{-1}(u) \in K_{c_{k}(\theta)}$, a contradiction. This shows $N_{c_{k}(\theta)}=\varnothing$. Therefore, by Remark II.3.12 in [13], there exists $\varepsilon_{0}>0$ such that for any $0<\varepsilon<\bar{\varepsilon} \leq \varepsilon_{0}$, there exists a continuous 1-parameter family of homeomorphisms $\eta(t, \cdot)$ of $S$, $0 \leq t<\infty$, with the properties: $\left(1^{0}\right) \eta(w, t)=w$, if $t=0$, or $\Psi^{\prime}(w)=0$, or $\left|\Psi(w)-c_{k}(\theta)\right| \geq \bar{\varepsilon}$; $\left(2^{0}\right) \Psi(\eta(w, t))$ is non-increasing in $t$ for any $w \in S ;\left(3^{0}\right) \eta\left(\Psi^{c_{k}(\theta)+\varepsilon}, 1\right) \subset \Psi^{c_{k}(\theta)-\varepsilon}$; $\left(4^{0}\right) \eta(\cdot, s) \circ \eta(\cdot, t)=\eta(\cdot, s+t)$ for all $s, t \geq 0 ; \quad\left(5^{0}\right) \eta(w, t)$ is odd in $w$ for $t \geq 0$.

Moreover, by $N_{c_{k}(\theta), \delta}=\varnothing$ we know that there exists $0<\varepsilon_{1}<\varepsilon_{0}$ such that $\Psi_{c_{k}(\theta)-\varepsilon_{1}}^{c_{k}(\theta)+\varepsilon_{1}} \cap K=\varnothing$.For each $w \in \Psi^{c_{k}(\theta)+\varepsilon_{1}}$, by the property $\left(3^{0}\right)$ of $\eta$ we know that $\Psi(\eta(w, 1)) \leq c_{k}(\theta)-\varepsilon_{1}$. Let $e=e(w)$ be the infimum of the time for which $\Psi(\eta(w, t)) \leq c_{k}(\theta)-\varepsilon_{1}$. It is easy to see that $e: \Psi^{c_{k}(\theta)+\varepsilon_{1}} \rightarrow[0,+\infty)$ is a continuous mapping. Since $\Psi$ is even, so is $e$. Define a mapping $h: \Psi^{c_{k}(\theta)+\varepsilon_{1}} \rightarrow \Psi^{c_{k}(\theta)-\varepsilon_{1}}$ by $h(w):=\eta(w, e(w))$. Then $h$ is odd and continuous. It follows from the mapping property of the genus and the definition of $c_{k}(\theta)$ that $k \leq \gamma\left(\Psi^{c_{k}(\theta)+\varepsilon_{1}}\right) \leq \gamma\left(\Psi^{c_{k}(\theta)-\varepsilon_{1}}\right) \leq k-1$ a contradiction. This completes the proof of Lemma 2.8.

Lemma 2.9 Let $\left\{\theta_{n}\right\} \subset(0,1]$ be such that $\theta_{n} \rightarrow 0$. Let $u_{n} \in E$ be a critical point of $J_{\theta_{n}}$ with $J_{\theta_{n}}\left(u_{n}\right) \leq c$ for some constant $c$ independent of $n$. Then, up to a subsequence, we have $u_{n} \stackrel{\text { weak }}{\longrightarrow} u$ in $H^{1}\left(\square^{N}\right), u_{n} \nabla u_{n} \stackrel{\text { weak }}{\longrightarrow} u \nabla u$ in $L^{2}\left(\square^{N}\right), u \in H^{1}\left(\square^{N}\right) \cap L^{\infty}\left(\square^{N}\right)$ is a critical point of $J$ and $J(u) \leq \liminf _{n \rightarrow \infty} J_{\theta_{n}}\left(u_{n}\right)$.

Proof. As the proof of Lemma 2.3 we can prove that the sequences $\left\{\left\|u_{n}\right\|_{H^{1}}\right\} \quad\left\{\left\|u_{n}\right\|_{22^{*}}\right\}$ $\left\{\int_{\square N}\left|u_{n}\right|^{2}\left|\nabla u_{n}\right|^{2} d x\right\}\left\{\int_{\square^{N}} G\left(x, u_{n}\right) d x\right\}$ and $\left\{\theta_{n}\left\|u_{n}\right\|_{W^{1,4}}^{4}\right\}$ are bounded. Hence, by the Sobolev embedding and the interpolation, up to a subsequence, one has $u_{n} \stackrel{\text { weak }}{\longrightarrow} u$ in $H^{1}\left(\square^{N}\right), u_{n} \rightarrow u$ in $L_{l o c}^{s}\left(\square^{N}\right.$ ) for $s \in\left[2,22^{*}\right), u_{n}(x) \rightarrow u(x)$ a.e. $x \in \square^{N}$. Up to a subsequence, $\lim _{n \rightarrow \infty} \int_{\square}\left|u_{n}-u\right|^{s} d x=0 \quad \forall s \in\left[2,22^{*}\right)$.

By using Moser iteration we can prove $u \in L^{\infty}\left(\square^{N}\right)$. Hence, by approximation we obtain $\left\langle J^{\prime}(u), \varphi\right\rangle=0, \forall \varphi \in H^{1}\left(\square^{N}\right) \cap L^{\infty}\left(\square^{N}\right)$. This shows that $u$ is a critical point of $J$. 
Moreover, one has $\quad \liminf _{n \rightarrow \infty} J_{\theta_{n}}\left(u_{n}\right) \geq \frac{a}{4} \int_{\square N}|\nabla u|^{2} d x+\frac{1}{4} \int_{\square N} V(x)|u|^{2} d x+\left(\frac{1}{4}-\frac{1}{22^{*}}\right) \int_{\square N}|u|^{22^{*}} d x$

$$
+\int_{\square}\left[\frac{1}{4} u g(x, u)-G(x, u)\right] d x=J(u)-\frac{1}{4}\left\langle J^{\prime}(u), u\right\rangle=J(u) .
$$

This completes the proof.

Proof of Theorem 1.1 Take a sequence $\left\{\theta_{n}\right\} \subset(0,1)$ with $\theta_{n} \downarrow 0$. Then for any fixed $k \in \square$, by Lemma 2.9 we know that there exists a sequence $\left\{v_{k}(n): n=1,2, \cdots\right\}$ of critical points for $\Psi$ such that $\Psi\left(v_{k}(n)\right)=c_{k}\left(\theta_{n}\right)$. Hence, for each $n \in \square$, Lemma 2.6 implies that $\left\{u_{k}(n):=m\left(v_{k}(n)\right)\right\}$ is a nontrivial critical point of $J_{\theta_{n}}$ and $J_{\theta_{n}}\left(u_{k}(n)\right)=c_{k}\left(\theta_{n}\right)$. Notice that $\delta_{1} \leq c_{k}\left(\theta_{n}\right) \leq c_{k}(1)$. Up to a subsequence, we have, as $n \rightarrow \infty, v_{k}(n) \rightarrow v_{k} u_{k}(n) \rightarrow u_{k}=m\left(v_{k}\right)$ in $H^{1}\left(\square^{N}\right), u_{k}(n) \nabla u_{k}(n) \rightarrow u_{k} \nabla u_{k}$ in $L^{2}\left(\square^{N}\right), \theta_{n} \int_{\square}\left(\left|\nabla u_{k}(n)\right|^{4}+\left|u_{k}(n)\right|^{4}\right) d x \rightarrow 0, J_{\theta_{n}}\left(u_{k}(n)\right) \rightarrow J\left(u_{k}\right):=c_{k}$ and $u_{k} \in H^{1}\left(\square^{N}\right) \cap L^{\infty}\left(\square^{N}\right)$ is a nontrivial critical point of $J$. By Lemma 2.6(3), $v_{k}$ is a critical point of $\Psi$.

Now, set $\tilde{K}:=\bigcup_{k=1}^{\infty} K_{c_{k}}$. Choose a subset $F$ of $\tilde{K}$ such that $F=-F$ and each orbit $O(u) \subset \tilde{K}$ has a unique representative in $F$. As proof of Lemma 2.11 in [15] we can prove that $F$ is an infinite set. Combining this with Lammas 2.6(3), we deduce Theorem 1.1.

\section{References}

[1] Jose F. L. Aires, Marco A.S. Souto, Existence of solutions for a quasilinear Schr ö dinger equation with vanishing potential, J. Math. Anal. Appl., 416 (2014) 924-946.

[2] M. Colin, L. Jeanjean, Solutions for a quasilinear Schr ö inger equation: a dual approach, Nonlinear Anal. 56 (2004), 213-226.

[3] J. M. do Q, U. Severo, Quasilinear Schrö dinger equations involving concave and convex nonlinearities, Commun. Pure Appl. Anal., 8 (2009) 621-644.

[4] X. D. Fang, A. Szulkin, Multiple solutions for a quasilinear Schrö dinger equation, J. Differential Equations, 254 (2013) 2015-2032.

[5] Z. H. Feng, X. Wu, H. X. Li, Multiple solutions for a modified Kirchhoff-type equation in $\square^{N}$, Math. Meth. Apple. Sci. (2014).

[6] E. Gloss, Existence and concentration of positive solutions for a quasilinear equation in $\square^{N}$, J. Math. Anal. Appl. 371 (2010) 465-484.

[7] P. L. Lions, The Concentration-compactness principle in the calculus of variations. The locally compact case, Part 2, Ann. Inst. H. Poincare Anal. Non Lineaire, 1 (1984), 223-283.

[8] A.G. Litvak, A.M. Sergeev, One dimensional collapse of plasma waves, JETP Lett. 27 (1978), 517-520.

[9] X. Q. Liu, J. Q. Liu, Z. Q. Wang, Quasilinear elliptic equations via perturbation method, Proc. Amer. Math. Soc., 141 (2013), 253-263.

[10] M. Poppenberg, On the local well posedness of quasi-linear Schrö inger equations in arbitrary space dimension, J. Diff. Eqns. 172 (2001), 83-115.

[11] M. Porkolab, M.V. Goldman, Upper hybrid solitons and oscillating two-stream instabilities, Phys. Fluids, 19 (1976), 872-881.

[12] David Ruiz, Gaetano Siciliano, Existence of ground states for a modified nonlinear Schrö dinger equation, Nonlinearity, 23 (2010), 1221-1233.

[13] M. Struwe, Variational Methods, Springer-Verlag, Berlin, second edition, 1996.

[14] M. B. Yang, Existence of solutions for a quasilinear Schrö dinger equation with subcritical nonlinearities, Nonlinear Anal., 75 (2012) 5362-5373.

[15] X. Wu, K. Wu, Geometrically distinct solutions for quasilinear elliptic equations, London Mathematical Society Nonlinearly 27 (2014) 987-1001. 